\section{Estilos de vida saludables: una ampliación de la mirada y su potencial en el marco de la pandemia}

\author{
ÁLVARO TALA ${ }^{1}$, EDUARDO VÁSQUEZ ${ }^{1}$, CECILIA PLAZA ${ }^{2}$
}

\section{Healthy lifestyles in times of pandemic}

SARS-CoV-2 pandemic generated a profound impact on people's health, emphasizing the relevance of healthy lifestyles. Recommendations on how to maintain adequate physical activity, diet, sleep and social connection have been issued. However, it is worth expanding our look to other possible elements related to lifestyles such as the relationship with technology, nature, pets and music. These areas should be included in the assessment and intervention from this perspective. To achieve changes, the values, beliefs, intentions, motivations, risk/benefit balances, capacity for self-regulation, previous history of changes and the person's sense of competence in relation to the possible changes that are being suggested, should be assessed. Individualized and contextualized suggestions that increase the intention of change should be made, avoiding confrontation and generalizations. Although there are still areas of uncertainty in this approach, particularly in relation to dosage and mechanisms of action, its development should be encouraged, given its great potential in terms of cost-effectiveness.

(Rev Med Chile 2020; 148: 1189-1194)

Key words: Behavior; Delivery of Health Care; Health; Life Style; Pandemics.
'Departamento de Psiquiatría y Salud Mental Norte, Facultad de Medicina, Universidad de Chile. Santiago, Chile.

${ }^{2}$ Unidad de Medicina Integrativa, Hospital Clínico de la Universidad de Chile. Santiago, Chile.

Esta investigación no contó con apoyo financiero.

Recibido el 14 de julio de 2020, aceptado el 11 de agosto de 2020.

Correspondencia a: Álvaro Tala Tajmuch Clínica Psiquiátrica Universitaria Universidad de Chile, Av. La Paz 1003, Santiago, Chile. alvarotalat@gmail.com

\section{$\mathrm{L}$}

os estilos de vida se han considerado durante gran parte de la historia de la humanidad como potenciales elementos de intervención para promover la salud de las personas. Estos se comprenden como las formas en las que viven sus vidas los individuos ${ }^{1}$. Tradicionalmente se han evaluado a nivel mundial y en Chile aquellos que se han ligado más directamente a la salud cardiovascular, como la nutrición, la actividad física, el sueño y el consumo de sustancias ${ }^{2-5}$, y en menor proporción, aquellos ligados a la salud mental como la conexión social ${ }^{1}$.

Hasta 70\% de las consultas en atención primaria en países desarrollados guardaría relación con enfermedades relacionadas a los estilos de vida. La comprensión del rol que estos factores juegan en la prevención de la morbilidad y la mortalidad asociada a múltiples enfermedades crónicas no transmisibles, junto con el bajo riesgo de intervenir desde esta mirada ${ }^{2}$, los ha convertido en un foco de particular interés en cuanto a la promoción y prevención en salud a nivel mundial y en nuestro país ${ }^{7}$. Además, cabe destacar que intervenciones desde esta perspectiva en general han demostrado ser costo-efectivas ${ }^{8-11}$.

La pandemia actual de SARS-CoV-2, ha generado un impacto global en la salud de las personas, no solo en relación con el daño directo dado por la infección del agente, sino también generado por las medidas implementadas necesarias para prevenir el contagio, como el distanciamiento físico y las cuarentenas. En este contexto de alta demanda emocional y física, limitado acceso a los sistemas de salud y escasos recursos, es que los estilos de vida saludables surgen como una oportunidad para contribuir a la salud que consideramos importante de atender.

En este sentido, han surgido múltiples recomendaciones de diferentes instituciones relevando una adecuada actividad física, dieta, sueño, 
conexión social y evitar el consumo de sustancias durante la pandemia, sin embargo, cobra importancia ampliar la mirada a otros posibles elementos relacionados con estilos de vida que han sido foco de investigación, que tradicionalmente no son evaluados y que se encuentran integrados en nuestra cotidianeidad, como potenciales intervenciones costo-efectivas y seguras en salud. Por otro lado, el contemplar los estilos de vida como parte de la estrategia, y revisar elementos de psicología de la conducta que se encuentra a la base de estos, podría ayudar a comprender mejor por qué algunas personas no han adherido adecuadamente a las recomendaciones para prevenir la transmisión del COVID-19 que implican cambios en sus estilos de vida como el lavado de manos frecuente y el uso de mascarilla, y cómo promover ese cambio.

Con el objetivo de ampliar la mirada de los estilos de vida que potenciarían la salud de las comunidades, a continuación, se describirán algunas áreas que forman parte de la rutina de muchas personas en la actualidad y que la evidencia científica ha avanzado lo suficiente como para afirmar que su adecuado análisis e indicación puede traer beneficios a la salud.

\section{Uso de la tecnología digital}

Actualmente las tecnologías son algo omnipresente en nuestra sociedad y nuestra rutina, sobre todo aquellas relacionadas con el mundo digital. La incorporación de tecnologías en la sociedad nos ha ayudado a modificar nuestro entorno, sin embargo, este proceso también ha modificado nuestra forma de vivir, entendernos y relacionarnos $^{12}$. A modo de ejemplo, ya existen en Chile más celulares que habitantes, y es habitual observar a la gente constantemente utilizando estos para comunicarse, informarse, jugar y revisar redes sociales. Este gran avance en la tecnología ha llevado incluso a la descripción de cuadros clínicos como el trastorno por uso de internet ${ }^{13} \mathrm{y}$ la depresión digital ${ }^{14}$. Es así como se ha podido observar como el uso de la tecnología en diversos patrones puede producir diferentes resultados en los individuos en la medida que se integran a su rutina ${ }^{15,16}$. El uso de la tecnología digital se ha disparado en el contexto de la pandemia, desde el teletrabajo, el telecolegio, la entretención de adultos y niños y la comunicación con otros, lo que lo convierte en un gran foco de posibles intervenciones para maximizar resultados favorables en salud. Por ejemplo, numerosas aplicaciones móviles se han basado en la gamificación, empleando por ejemplo un sistema de puntos, retroalimentación del progreso, insignias y misiones, con el objetivo de desarrollar cambios a favor del bienestar ${ }^{17}$.

\section{Interacción con la naturaleza}

Nos hemos relacionado con la naturaleza desde el principio de nuestra existencia como especie y lo hacemos en mayor o menor medida a lo largo de toda nuestra vida. Pese a esto, la mirada científica aplicada a esta relación es relativamente reciente. Estudios han mostrado que la exposición a ambientes naturales en diferentes formas, incluyendo la exposición pasiva, o directa mediante aventuras, caminatas $\mathrm{u}$ otras actividades intencionadas ${ }^{18}$, podría traer beneficios a la salud tanto desde elementos tan concretos como la regulación de variables fisiológicas relacionadas con la salud cardiovascular, hasta elementos más subjetivos como podrían ser la sensación de bienestar y las relaciones interpersonales ${ }^{19}$. Incluso se ha asociado con una reducción de la mortalidad por enfermedad cardiovascular ${ }^{20}$. Esto posiblemente mediante mecanismos como el aumento de actividad física, la inducción de una respuesta de relajación, exposición a aire más limpio o promover capital social ${ }^{21}$, entre otros posibles mecanismos.

Actualmente la mitad de la población mundial vive en áreas urbanas y se estima en las próximas décadas el porcentaje aumente a $70 \%{ }^{22}$. El hecho de que las personas en contextos urbanos tienden a pasar hasta $90 \%$ de su tiempo puertas adentro, lo cual en el contexto de la pandemia probablemente ha aumentado, podría llevar a pensar que este no sería un ámbito relevante en nuestro contexto actual, sin embargo, se ha visto que un entorno más verde en espacios cerrados o bien el observar entornos verdes por ventanas, produciría beneficios en áreas como el bienestar psicológico, estrés percibido, niveles de ansiedad, la productividad y parámetros psicofisiológicos, lo que podría explicarse a través de mecanismos como la regulación de la temperatura, humedad y niveles de $\mathrm{CO}_{2}$ en el ambiente intradomiciliario ${ }^{23}$. En este sentido se han realizado estudios con exposición a plantas específicas por un tiempo determinado, con resul- 
tados promisorios, aunque sin duda requieren ser replicados, para en el futuro lograr una adecuada prescripción de naturaleza basada en la evidencia $^{24-26}$. La jardinería doméstica ha sido estudiada especialmente en adultos mayores, encontrando que favorece el envejecimiento exitoso, destacando entre sus beneficios un menor riesgo de caídas y mayor sensación de satisfacción, sentido de competencia y realización personal ${ }^{27}$. En busca de alternativas a la experiencia in vivo de naturaleza, se ha encontrado también que la exposición a la naturaleza mediante realidad virtual podría desempeñar un rol en contribuir al bienestar físico y emocional ${ }^{18}$. Finalmente, también se ha observado que el sentir una mayor conexión con la naturaleza ya podría ser suficiente para tener mejores resultados en términos de bienestar eudaimónico ${ }^{28}$. Estos hallazgos podrían tenerse en consideración durante el diseño de las ciudades e incluso en el de los ambientes trabajo.

\section{Relación con mascotas}

Los animales han acompañado desde hace siglos al hombre y han contribuido enormemente a su desarrollo. Dentro de este acompañamiento, existen las mascotas, entendidas como animales de compañía con los cuales se comparte un vínculo, que suelen integrar la rutina cotidiana de sus dueños y que con el avance de la sociedad se han hecho cada vez más frecuentes y diversas. En Chile, según datos de Cadem, 73\% de las personas tiene al menos una mascota, con un promedio de dos por hogar ${ }^{29}$. Estas se han asociado a una serie de beneficios, como el promover cambios hacia conductas más saludables de alimentación y actividad física, aumentar el capital social y las redes de apoyo, disminuir la probabilidad de conductas autodestructivas, niveles de sintomatología ansioso-depresiva y la sensación de soledad, pudiendo incluso el solo acto de acariciar una mascota disminuir niveles de presión arterial y concentraciones de colesterol ${ }^{30,31}$. Si bien se desconocen los mecanismos subyacentes específicos que median estos efectos positivos, se ha encontrado que la interacción con las mascotas aumenta los niveles de beta-endorfina, oxitocina, prolactina y dopamina; y disminuye la concentración de cortisol ${ }^{32}$. En este sentido, podrían ser particularmente útiles en el contexto actual donde el contacto físico con otras personas se encuentra limitado, e incluso podrían ayudar al sistema inmune frente al COVID-19 mediante inmunización cruzada ${ }^{33}$, considerando además que no se ha encontrado que los animales de compañía aumenten el riesgo de contagio de COVID- $19^{34}$. A pesar de sus beneficios, también se han descrito efectos adversos, tanto médicos, por ejemplo, alergias y zoonosis ${ }^{31}$; como psicológicos, por ejemplo, emociones negativas cuando el animal se lastima, o el proceso de duelo cuando muere $^{32}$. Se debe tener presente que la mayor parte de la investigación con mascotas se ha centrado en la tenencia de perros, y en menor medida, gatos. Poco se sabe sobre los efectos beneficiosos de otras mascotas, incluidas las exóticas e incluso animales robóticos. Estos últimos podrían ser especialmente útiles en personas que, debido a problemas de salud, financieros o disponibilidad de tiempo, no pueden hacerse cargo de una mascota ${ }^{32}$.

\section{Los sonidos y la música que nos rodean}

En el mundo moderno el silencio es casi un lujo. Incluso en aquellos espacios o tiempos donde es posible, la tecnología ha facilitado el acceso a los sonidos y la música de forma sencilla, por lo que con frecuencia las personas optan por utilizarlos. Existe amplia literatura sobre el potencial de estas variables como intervenciones en salud en diversos contextos, como residencias y hospitales, mediante su impacto en aspectos relacionados tanto con la salud mental, como el evocar la paz y tranquilidad, como con salud física en ámbitos como la variabilidad de la frecuencia cardiaca y la actividad electrodermal, sobre todo cuando se reemplazan sonidos excesivos o aversivos por otros más adecuados ${ }^{35-38}$. En este sentido, sobre todo en ambientes urbanos, existe un gran potencial en el uso intencionado de los sonidos y la música para contribuir al bienestar de las personas en el contexto de la pandemia y en particular del personal de salud en su lugar de trabajo.

\section{Orientaciones sobre cómo generar cambios en estilos de vida en pandemia}

En general la adherencia a las indicaciones farmacológicas, entendida como el grado en que la conducta de la persona coincide con la indica- 
ción, tiende a alcanzar cerca de $50 \%{ }^{39}$, lo que se ha descrito que podría disminuir hasta $70 \%$ para intervenciones en estilos de vida ${ }^{40}$. Esta mala adherencia probablemente ha sido exacerbada en el contexto pandémico actual, dadas las limitaciones existentes para poder acceder a los servicios de salud y realizar actividades.

Considerando estos problemas de adherencia, se han intentado aplicar a la clínica múltiples teorías de cambio con la intención de aumentar la probabilidad de que las personas inicien y sostengan cambios en sus estilos de vida, siendo las principales teorías el modelo de creencias de salud, la teoría de la conducta planificada, el modelo de las etapas del cambio, la teoría de la autodeterminación y la teoría de la auto regulación temporal, no siendo ninguno categóricamente superior a otro, pero compartiendo elementos comunes que pueden orientar a los clínicos sobre cómo introducir cambios en estilos de vida ${ }^{41,42}$. Estas teorías se enfocan en la importancia de indagar sobre los valores, creencias, intenciones, motivaciones, balances riesgo/beneficio, capacidad de auto regulación, historia previa de cambios y el sentido de competencia que tiene la persona en relación a los posibles cambios que se le están sugiriendo, junto con el momento en que este se está planteando dicho cambio, para desde ahí ir generando sugerencias específicas, individualizadas y contextualizadas que vayan en línea con la persona y aumenten su intención de cambio. Esto mediante con preguntas habitualmente abiertas, no juiciosas, como "¿cuáles serían los pros y los contras del cambio?”, “ ¿cómo responderían tus cercanos a estos cambios?", “¿qué pasaría si el cambio no ocurre?" o “¿qué podrían hacer otras personas que conoces en tu situación?"41. Múltiples estudios han mostrado que esto puede realizarse mediante intervenciones online, en diversas poblaciones etarias, y que posiblemente es efectivo, sobre todo si las intervenciones son multicomponente ${ }^{43-46}$, lo cual sería una ventaja en el marco de la pandemia, facilitando el acceso a intervenciones en salud y eventualmente podría ser una ventana de oportunidad para mejorar la adherencia a las medidas de higiene recomendadas por la autoridad.

También vale la pena tener en consideración dos situaciones frecuentes, que es deseable evitar cuando se hacen sugerencias de estilos de vida saludables, que se desprenden de los principios mencionados anteriormente. La primera es intentar imponer un cambio. Un ejemplo de esta situación podría ser una persona con diabetes que no adhiere a las medidas no farmacológicas que ayudan a regular su glicemia, y su tratante opta por enfocar el problema mediante comentarios como "si usted no sigue $\mathrm{X}$, va a terminar ciego y amputado". Este abordaje confrontacional y autoritario no suele ser el más efectivo en términos de producir cambios $^{47}$, ya que no considera la individualidad de las personas. La segunda, tiene que ver con la tendencia a hacer recomendaciones generales, sin considerar las variables individuales que pueden modificar el resultado de dicha recomendación en el individuo, pudiendo ser el resultado incluso peor a lo existente previo a la recomendación. Por ejemplo, el recomendar evitar el ejercicio en la tarde noche para promover el buen dormir puede ser una indicación que prive de una estrategia de regulación emocional efectiva a alguien que lo realiza habitualmente, sobre todo teniendo en consideración de que la evidencia de que el ejercicio a esa hora altera el sueño no es sólida y no se suele considerar los efectos moderadores del tipo de ejercicio, duración, intensidad, nivel de acondicionamiento físico de la persona, edad, sexo, entre otros ${ }^{48}$.

\section{Perspectivas a futuro}

Los potenciales focos por abordar desde la perspectiva de los estilos de vida saludables mencionados en este artículo no pretenden ser abarcativos de todas las posibilidades, existiendo otros ámbitos como la expresión creativa y la espiritualidad por mencionar algunos. Más bien se pretende invitar a los profesionales de la salud a ampliar el marco de acción de este enfoque y entregar más herramientas que puedan contribuir a la salud de las personas en el contexto actual.

A su vez, se debe considerar que aún existe un gran desafío en esta disciplina en cuanto a las posologías específicas de las diversas áreas mencionadas. Por ejemplo, cuáles serían los mejores sonidos y músicas en términos de frecuencia de onda, volumen, melodía, duración que producirían los mejores resultados en salud ${ }^{49}$, o cuál sería la mejor cantidad, ubicación y tipo de plantas de interior ${ }^{23}$, sin embargo, esto debiese ser un estímulo para su mayor desarrollo, dado el gran potencial que 
poseen en términos de costo-efectividad a nivel de salud poblacional.

\section{Referencias}

1. Zaman R, Hankir A, Jemni M. Lifestyle factors and mental health. Psychiatr Danub. 2019; 31 (3): 217-20.

2. Petrides J, Collins P, Kowalski A, Sepede J, Vermeulen M. Lifestyle Changes for Disease Prevention. Prim Care Clin Off Pract 2019; 46 (1): 1-12.

3. Claas SA, Arnett DK. The role of healthy lifestyle in the primordial prevention of cardiovascular disease. Curr Cardiol Rep 2016; 18 (6): 56.

4. Leiva AM, Petermann-Rocha F, Martínez-Sanguinetti MA, Troncoso-Pantoja C, Concha Y, Garrido-Méndez A, et al. Asociación de un índice de estilos de vida saludable con factores de riesgo cardiovascular en población chilena. Rev Med Chile 2018; 146: 1405-14.

5. Vásquez-Gómez JA, Matus-Castillo C, Petermann-Rocha F, Concha-Cisternas Y, Leiva AM, Martínez-Sanguinetti MA, et al. Caracterización de los estilos de vida en dueñas de casa chilenas. Análisis de la Encuesta Nacional de Salud 2009-2010. Rev Med Chile 2019; 147: 1144-53.

6. Egger GJ, Binns AF, Rossner SR. The emergence of "lifestyle medicine" as a structured approach for management of chronic disease. Med J Aust 2009; 190 (3): 143-5.

7. Troncoso-Pantoja C, Martínez-Sanguinetti MA, Ulloa $\mathrm{N}$, Celis-Morales C. La mayoría de las enfermedades cardiovasculares se atribuyen a factores de riesgo que podrían ser modificados con cambios de los estilos de vida. Rev Med Chile 2020; 148: 126-8.

8. White P, Skirrow H, George A, Memon A. A systematic review of economic evaluations of local authority commissioned preventative public health interventions in overweight and obesity, physical inactivity, alcohol and illicit drugs use and smoking cessation in the United Kingdom. J Public Health (Bangkok) 2018; 40 (4): e52130 .

9. Sun Y, You W, Almeida F, Estabrooks P, Davy B. The effectiveness and cost of lifestyle interventions including nutrition education for diabetes prevention: a systematic review and meta-analysis. J Acad Nutr Diet 2017; 117 (3): 404-21.

10. Alouki K, Delisle H, Bermúdez-Tamayo C, Johri M. Lifestyle interventions to prevent type 2 diabetes: a systematic review of economic evaluation studies. J Diabetes Res 2016; 2016: 2159890.

11. Sutton L, Karan A, Mahal A. Evidence for cost-effectiveness of lifestyle primary preventions for cardiovascular disease in the Asia-Pacific Region: a systematic review.
Global Health 2014; 10 (1): 79.

12. Grande M, Cañón R, Cantón I. Tecnologías de la información y la comunicación: Evolución del concepto y características. Int J Educ Res Innov 2016; 6: 218-30.

13. Montag C, Reuter M. Internet addiction. Springer; 2017. 143-150 p.

14. Ghaemi SN. From Research to Clinical Practice Digital depression: a new disease of the millennium? Acta Psychiatr Scand 2020; 1: 1-6.

15. Canadian Paediatric Society Ottawa, Ontario DHTF. Digital media: Promoting healthy screen use in schoolaged children and adolescents. Paediatr Child Health 2019; 24 (6): 402-8.

16. Tala Á, Vásquez E. Conexión en tiempos de COVID-19. Rev Med Chile 2020; 148: 557-8.

17. Cheng VWS, Davenport T, Johnson D, Vella K, Hickie IB. Gamification in apps and technologies for improving mental health and well-being: Systematic review. J Med Internet Res 2019; 21 (6): 1-15.

18. White MP, Yeo NL, Vassiljev P, Lundstedt R, Wallergård $\mathrm{M}$, Albin $\mathrm{M}$, et al. A prescription for "nature" - The potential of using virtual nature in therapeutics. Neuropsychiatr Dis Treat 2018; 14: 3001-13.

19. Mygind L, Kjeldsted E, Hartmeyer RD, Mygind E, Bølling M, Bentsen P. Immersive nature-experiences as health promotion interventions for healthy, vulnerable, and sick populations? A systematic review and appraisal of controlled studies. Front Psychol 2019; 10: 943.

20. WHO Regional Office for Europe. Urban green spaces and health 2016; 92.

21. Frumkin H, Bratman GN, Breslow SJ, Cochran B, Kahn Jr PH, Lawler JJ, et al. Nature contact and human health: A research agenda. Environ Health Perspect 2017; 125 (7): 75001.

22. Nieuwenhuijsen MJ, Khreis H, Triguero-Mas M, Gascon M, Dadvand P. Fifty shades of green. Epidemiology 2017; 28 (1): 63-71.

23. Deng L, Deng Q. The basic roles of indoor plants in human health and comfort. Environ Sci Pollut Res 2018; 25 (36): 36087-101.

24. Igarashi M, Miwa M, Ikei H, Song C, Takagaki M, Miyazaki Y. Physiological and psychological effects of viewing a kiwifruit (Actinidia deliciosa 'Hayward') orchard landscape in summer in Japan. Int J Environ Res Public Health 2015; 12 (6): 6657-68.

25. Ikei H, Komatsu M, Song C, Himoro E, Miyazaki Y. The physiological and psychological relaxing effects of viewing rose flowers in office workers. J Physiol Anthropol 2014; 33 (1): 1-5.

26. Li ZM, Liu H, Zhang WZ, Liu H. Psychophysiological and cognitive effects of strawberry plants on people in 
isolated environments. J Zhejiang Univ Sci B 2020; 21 (1): 53-63.

27. Gagliardi C, Piccinini F. The use of nature - based activities for the well-being of older people: An integrative literature review. Arch Gerontol Geriatr 2019; 83 (December 2018): 315-27.

28. Martin L, White MP, Hunt A, Richardson M, Pahl S, Burt J. Nature contact, nature connectedness and associations with health, wellbeing and pro-environmental behaviours. J Environ Psychol 2020; 68: 101389.

29. Cadem. El Chile que viene - Mascotas [Internet]. 2019. 2020 [cited $2020 \mathrm{Jul}$ 8]. Available from: https://www. cadem.cl/encuestas/el-chile-que-viene-mascotas/

30. Hodgson K, Barton L, Darling M, Antao V, Kim FA, Monavvari A. Pets' impact on your patients' health: leveraging benefits and mitigating risk. J Am Board Fam Med 2015; 28 (4): 526-34.

31. Friedman E, Krause-Parello CA. Companion animals and human health: benefits, challenges, and the road ahead for human-animal interaction. Rev Sci Tech 2018; 37 (1): 71-82.

32. Matchock RL. Pet ownership and physical health. Curr Opin Psychiatry. 2015; 28 (5): 386-92.

33. Jurgel J, Filipiak KJ, Szarpak $€$, Jaguszewski M, Smerka J, Dzieciątkowski T. Do pets protect their owners in the COVID-19 era? Med Hypotheses. 2020; 142: 109831.

34. Tiwari R, Dhama K, Sharun K, Iqbal Yatoo M, Malik YS, Singh R, et al. COVID-19: animals, veterinary and zoonotic links. Vet Q 2020; 2176 (May): 1-22.

35. Iyendo TO. Exploring the effect of sound and music on health in hospital settings: A narrative review. Int J Nurs Stud 2016; 63: 82-100.

36. Erfanian M, Mitchell AJ, Kang J, Aletta F. The Psychophysiological Implications of Soundscape: A Systematic Review of Empirical Literature and a Research Agenda. Int J Environ Res Public Health. 2019; 16 (19): 3533.

37. Mileski M, Brooks M, Kirsch A, Lee F, LeVieux A, Ruiz A. Positive physical and mental outcomes for residents in nursing facilities using music: a systematic review. Clin Interv Aging 2019; 14: 301.

38. Oleksy AJ, Schlesinger JJ. What's all that noise-Improving the hospital soundscape. J Clin Monit Comput 2019; 33 (4): 557.

39. Nieuwlaat R, Wilczynski N, Navarro T, Hobson N, Jeffery R, Keepanasseril A, et al. Interventions for enhancing medication adherence. Cochrane database Syst
Rev 2014; (11).

40. Stonerock GL, Blumenthal JA. Role of counseling to promote adherence in healthy lifestyle medicine: strategies to improve exercise adherence and enhance physical activity. Prog Cardiovasc Dis 2017; 59 (5): 455-62.

41. Barley E, Lawson V. Using health psychology to help patients: theories of behaviour change. Br J Nurs 2016; 25 (16): 924-7.

42. McDermott MS, Oliver M, Iverson D, Sharma R. Effective techniques for changing physical activity and healthy eating intentions and behaviour: A systematic review and meta-analysis. Br J Health Psychol 2016; 21 (4): 827-41.

43. Rose T, Barker M, Jacob CM, Morrison L, Lawrence $\mathrm{W}$, Strömmer S, et al. A systematic review of digital interventions for improving the diet and physical activity behaviors of adolescents. J Adolesc Heal 2017; 61 (6): 669-77.

44. Aalbers T, Baars MAE, Rikkert MGMO. Characteristics of effective Internet-mediated interventions to change lifestyle in people aged 50 and older: a systematic review. Ageing Res Rev 2011; 10 (4): 487-97.

45. Beleigoli AM, Andrade AQ, Cançado AG, Paulo MNL, Maria De Fátima HD, Ribeiro AL. Web-based digital health interventions for weight loss and lifestyle habit changes in overweight and obese adults: systematic review and meta-analysis. J Med Internet Res 2019; 21 (1): e298.

46. Kohl LFM, Crutzen R, de Vries NK. Online prevention aimed at lifestyle behaviors: a systematic review of reviews. J Med Internet Res 2013; 15 (7): e146.

47. McNeil DW, Addicks SH, Randall CL. Motivational Interviewing and Motivational Interactions for Health Behavior Change and Maintenance [Internet]. Oxford University Press; 2017. Available from: https://www.oxfordhandbooks.com/ view/10.1093/oxfordhb/9780199935291.001.0001/ oxfordhb-9780199935291-e-21.

48. Irish LA, Kline CE, Gunn HE, Buysse DJ, Hall MH. The role of sleep hygiene in promoting public health: A review of empirical evidence. Sleep Med Rev 2015; 22: 23-36.

49. Martin-Saavedra JS, Vergara-Méndez LD, Pradilla I, Vélez-van-Meerbeke A, Talero-Gutiérrez C. Standardizing music characteristics for the management of pain: A systematic review and meta-analysis of clinical trials. Complement Ther Med 2018; 41: 81-9. 